\title{
RULE-BASED EQUATION FOR ELASTIC TRIPPING ANALYSIS OF ANGLE-BAR STIFFENERS
}

\author{
A. Rahbar-Ranji
}

Department of Ocean Engineering, AmirKabir University, Hafez Ave. 15914, Tehran, Email: rahbar@aut.ac.ir

\begin{abstract}
:
Tripping is one of buckling modes in stiffened plates which could be occurred in the stiffeners with high flexural rigidity and low torsional rigidity. Rule-base expressions for calculation of sectorial moment of inertia of angle-bar stiffeners are scattered. An expression for calculation of sectorial moment of inertia of angle-bar stiffeners is derived based on energy method and beam theory. Sectorial moment of inertia of different angle-bar stiffeners are calculated and compared with the values calculated by different classification society rules. It is found that some of the rule-based equations for calculation of sectorial moment of inertia of angle-bar stiffeners are inaccurate. Euler tripping stress of different angle bars are calculated by energy method and compared with rulebased equation and finite element method. It is found that, rule-based expression for calculation of polar moment of inertia of angle-bar stiffeners neglects one term, which could lead up to 10\% overestimation of Euler tripping stress.
\end{abstract}

Keywords: Angle bar stiffener, sectorial moment of inertia, stiffened plate, tripping.

\section{Introduction}

Stiffened plate consists of thin plate stiffened with welded longitudinal stiffeners and transverse girders. Stiffeners are closely spaced beams supported by stronger widely spaced transverse girders (see Fig. 1).

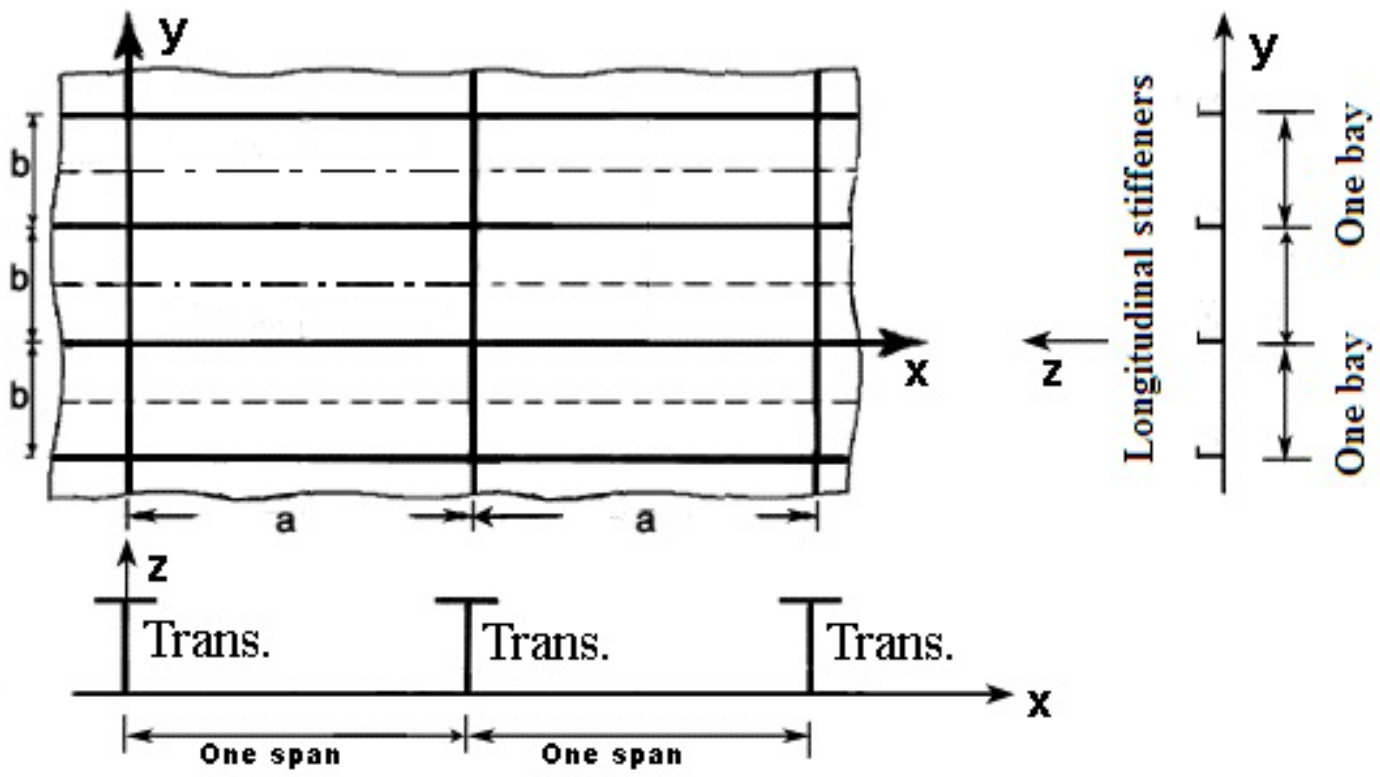

Fig. 1: Description of stiffened plate and coordinate system

The primary function of stiffened plates is to support lateral pressures imposed from weight of cargo or sea pressure. However, bending of ship hull girder induces in-plane loading as well. Due to this in-plane stresses, buckling of stiffened plates could be occurred. Depending upon dimensions of stiffeners and attached plate, different modes of buckling including plate buckling, stiffener buckling and interaction of them occurs. Torsional buckling or tripping (Fig. 2) is one of the buckling modes in stiffened plates in which stiffener rotates 
as a rigid body about intersection point of stiffener to attached plate. This mode occurs in the stiffeners with high flexural rigidity and low torsional rigidity (Danielson et al. 1990).

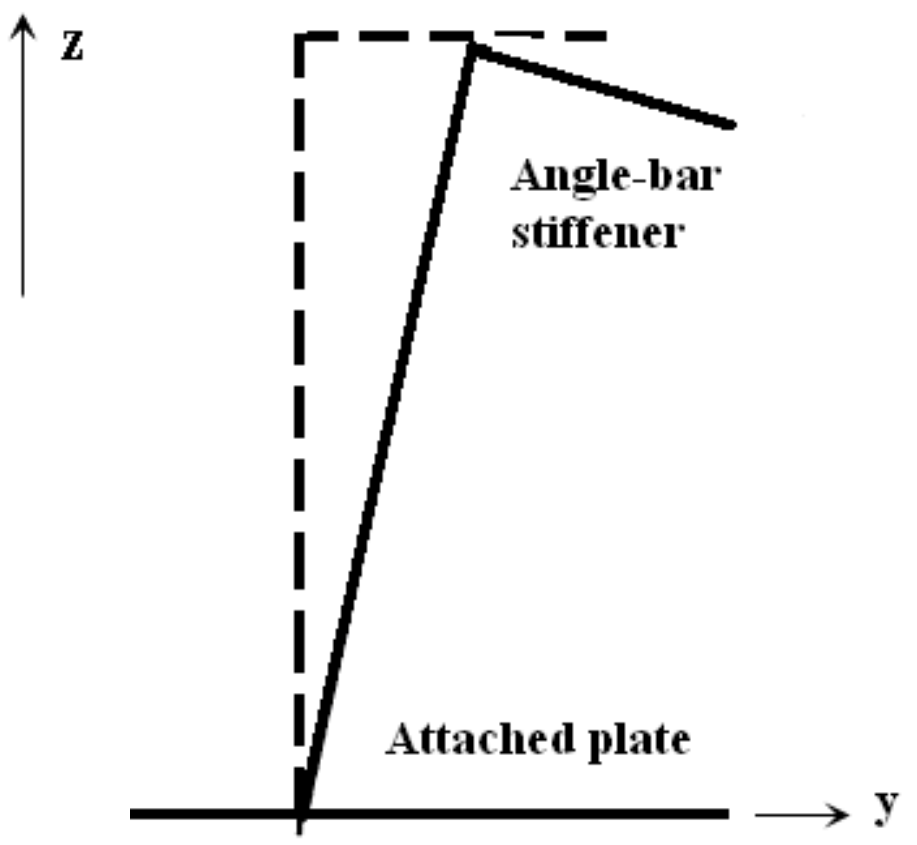

Fig. 2: Tripping or torsional buckling mode in stiffened plate

Surprisingly little material exists in the literature on the subject of the torsional instability of stiffeners welded to continuous plating (Danielson et al. 1990). Timoshenko and Gere (1961) have developed basic equation for torsional buckling analysis of any thin-walled beams. Danielson et al. (1990) proposed a mathematical model based on energy method to analyze elastic tripping stress of thin-walled beams. Rahbar-Ranji (2012) has used energy method to analyze Euler tripping stress of asymmetric stiffeners in stiffened plate. He has proposed a strain distribution for sideways bending based on normalized warping function and has used plate theory for out-of-plane deflection.

Classification societies have been provided simple formula for evaluation of Euler tripping stress of stiffeners in stiffened plates. In this formula sectorial moment of inertia plays an important role. Given equation for calculation of sectorial moment of inertia for angle-bar stiffeners in rules are scattered. It is the main aim of the present work to investigate the accuracy and applicability of these formulas. An expression for sectorial moment of inertia for angle-bar stiffeners is derived based on energy method (Rahbar-Ranji 2012) and confirmed by beam theory. This expression is used to investigate accuracy and applicability of rule-based equations for calculation of sectorial moment of inertia. Euler tripping stress of different angle-bar stiffeners are calculated and compared with finite element method (FEM) and rule-based equation.

\section{Basic Equations}

Equilibrium method or energy approach can be employed for buckling analysis of structures. Rahbar-Ranji (2012) has used energy method for calculating Euler tripping stress of angle-bar stiffeners. He has proposed a strain distribution for sideways bending based on normalized warping function and has used plate theory for out-of-plane deformation of the web and flange. Euler tripping stress of angle bar stiffeners is calculated from following equation (Rahbar-Ranji 2012),

$$
\sigma_{E T}=\frac{\left[\frac{\pi^{4} E t_{w}^{3} h_{w}}{144\left(1-v^{2}\right) a^{3}}+\frac{\pi^{2} E t_{w}^{3}}{24(1+v) a h_{w}}\right]+\left[\frac{\pi^{4} E t_{f}^{3} b_{f}^{3}}{144\left(1-v^{2}\right) a^{3} h_{w}^{2}}+\frac{\pi^{2} E t_{f}^{3} b_{f}}{24(1+v) a h_{w}^{2}}\right]+\frac{\pi^{4} E I_{z f}}{4 a^{3}}}{\left[\frac{\pi^{2} t_{w} h_{w}}{12 a}\right]+\left[\frac{\pi^{2} t_{f} b_{f}^{3}}{12 a h_{w}^{2}}+\frac{\pi^{2} t_{f} b_{f}}{4 a}\right]}
$$


where $E$ is Young's modulus, $v$ is Poisson's ratio, $h_{w}$ is the web height, $t_{w}$ is the web thickness, $b_{f}$ is the flange width, $t_{f}$ is the flange thickness, $a$ is the length of the angle bar stiffener. The loaded edges are taken to be simply supported and $I_{z f}$ is the moment of inertia of cross-section about vertical axis ( $z$ axis in Fig. 3) and given as follows (Rahbar-Ranji 2012):

$$
I_{z f}=h_{w} t_{w} y_{0}^{2}+\frac{b_{f}^{3} t_{f}}{12}+b_{f} t_{f}\left(\frac{b_{f}}{2}-y_{0}\right)^{2}
$$

where $y_{0}$ is the position of vertical neutral axis in respect to web of angle bar (Fig. 3), and calculated as follows:

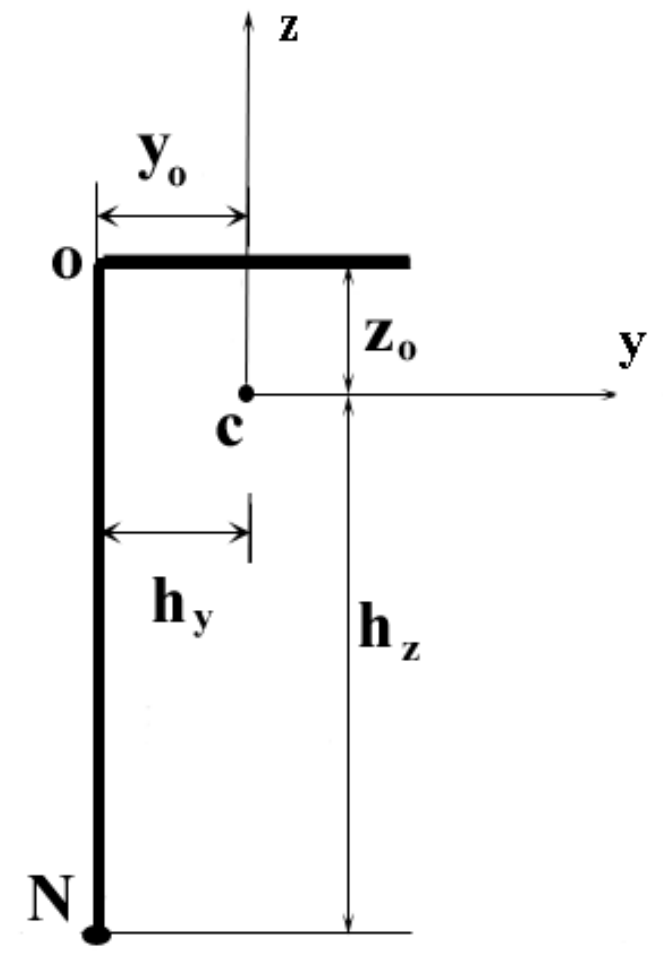

Fig. 3: Geometry and coordinate system of angle bar

$$
y_{0}=\frac{b_{f}^{2} t_{f}}{2\left(h_{w} t_{w}+b_{f} t_{f}\right)}
$$

If the ratios of $\frac{b_{f}^{2}}{a^{2}}$ and $\frac{h_{w}^{2}}{a^{2}}$ be small enough, then the corresponding terms in Equation (1) can be omitted, leading to following equation for calculation of the Euler tripping stress of angle-bar stiffeners in stiffened plate:

$$
\sigma_{E T}=\frac{G\left(\frac{t_{w}^{3} h_{w}}{3}+\frac{t_{f}^{3} b_{f}}{3}\right)+E I_{z f} h_{w}^{2} \frac{\pi^{2}}{a^{2}}}{\frac{t_{w} h_{w}^{3}}{3}+\frac{t_{f} b_{f}^{3}}{3}+t_{f} b_{f} h_{w}^{2}}
$$

For brevity, Equation (4) is expressed as follows:

$$
\sigma_{E T}=\frac{G J+E I_{W N} \frac{\pi^{2}}{a^{2}}}{I_{N}}
$$


where $G$ is shear modulus, $J$ is Saint Venant's moment of inertia, $I_{N}$ is the polar moment of inertia about point $\mathrm{N}$ and $I_{W N}$ is the sectorial moment of inertia about point $\mathrm{N}$, and are defined as follows:

$$
\begin{aligned}
& I_{N}=\frac{t_{w} h_{w}^{3}}{3}+\frac{t_{f} b_{f}^{3}}{3}+t_{f} b_{f} h_{w}^{2} \\
& J=\frac{t_{w}^{3} h_{w}}{3}+\frac{t_{f}^{3} b_{f}}{3} \\
& I_{W N}=h_{w}^{2} I_{z f}
\end{aligned}
$$

Substituting Equation (2) into (8) and manipulating it, leads to the following expression for calculation of sectorial moment of inertia of angle-bar stiffener about point $\mathrm{N}$ :

$$
I_{W N}=\frac{t_{f} b_{f}^{3} h_{w}^{2}}{12} \frac{4 t_{w} h_{w}+t_{f} b_{f}}{t_{w} h_{w}+t_{f} b_{f}}
$$

Timoshenko and Gere (1961) have developed basic equation for calculation of Euler stress in torsional buckling of an open thin-walled beam based on beam theory when centroid and shear center coincide as follows:

$$
\sigma_{E T}=\frac{G J+E I_{W} \frac{\pi^{2} m^{2}}{a^{2}}}{I_{o}}
$$

where $I_{W}$ is the sectorial moment of inertia about shear center, $J$ is Saint Venant's moment of inertia, $I_{o}$ is the polar moment of inertia about shear center, and $m$ is the number of half waves along the beam which is assumed as one in order to have minimum buckling stress. When torsion is enforced about a point say, N, Equation (10) is modified as follows:

$$
\sigma_{E T}=\frac{G J+E\left(I_{y}\left(y_{o}-h_{y}\right)^{2}+I_{z}\left(z_{o}-h_{y}\right)^{2}+I_{W}\right) \frac{\pi^{2}}{a^{2}}}{I_{o}+A\left(h_{y}^{2}+h_{z}^{2}-y_{o}^{2}-z_{o}^{2}\right)}
$$

where $y_{o}$ and $z_{o}$ are the coordinates of shear center with respect to centroid, $h_{y}$ and $h_{z}$ are the coordinates of point $\mathrm{N}$ with respect to centroid (Fig. 3), $A$ is the total cross-sectional area, and $I_{y}$ and $I_{z}$ are moment of inertia of cross section about axes $y$ and $z$, respectively. The polar moment of inertia about shear center can be written as follows:

$$
I_{o}=I_{C}+A\left(y_{o}^{2}+z_{o}^{2}\right)
$$

where $I_{C}$ is the polar moment of inertia about centroid. In the same way, polar moment of inertia about point $\mathrm{N}$ is expressed as follows:

$$
I_{N}=I_{C}+A\left(h_{y}^{2}+h_{z}^{2}\right)
$$

or

$$
I_{N}=I_{o}+A\left(h_{y}^{2}+h_{z}^{2}\right)-A\left(y_{o}^{2}+z_{o}^{2}\right)
$$

Timoshenko and Gere (1961) had derived Equation (11) when $y$ and $z$ are the principal axes, however this equation can be used for any other axes with its origin at centroid. If these axes are taken parallel to web and flange of angle bar (Fig. 3) then following relations are exist,

$$
\begin{aligned}
& y_{o}-h_{y}=0 \\
& z_{o}-h_{z}=h_{w}
\end{aligned}
$$


Warping occurs in torsion of all bars, except circular one. The warping function in an open thin-walled beam is calculated as follows (Timoshenko and Gere, 1961):

$\omega_{D}(s)=\int_{s} \rho d s$

where, $s$ is distance along the middle line of cross section from arbitrary reference point, and $\rho$ is normal component of position vector at point $s$ from center of torsion. In torsion of prismatic members the shape of the warping is the same as the warping function. The net warping distribution or net warping function should be zero over cross section, since the beam is under pure torsion. The normalized warping function is defined as follows:

$\omega_{N}(s)=\bar{\omega}_{D}-\omega_{D}(s)$

where, $\bar{\omega}_{D}$ is the average value of warping function, $\omega_{D}(\mathrm{~s})$.

Sectorial moment of inertia is defined as follows (Timoshenko and Gere 1961):

$I_{W}=\int_{m}\left(\omega_{N}(s)\right)^{2} t(s) d s$

where, $m$ is the length of the middle line of the cross section. Sectorial moment of inertia of an angle-bar about its shear center is equal to zero. Substituting Equations (14), (15) and (16) into (11) leads to following expression for calculation of tripping Euler stress of angle-bar stiffeners about point N:

$\sigma_{E T}=\frac{G J+E I_{z} h_{w}^{2} \frac{\pi^{2}}{a^{2}}}{I_{N}}$

It can be seen that Equation (5) based on energy method and Equation (20) based on beam theory are exactly identical, since Equation (2) has the same meaning as moment of inertia about neutral axis $z$, in other words, $I_{z f}$ and $I_{\mathrm{z}}$ are the same. Therefore, Equation (8) for calculation of sectorial moment of inertia of an angle-bar stiffener about junction point of stiffener which is derived from energy method has confirmed by beam theory.

\section{Tripping Analysis of Angle-bar Stiffeners by Rule-based Equation}

In all classification societies given equation for calculation of Euler tripping stress is in the following form (ABS 2004, BV 2002, CSR 2006, DnV 2010, GL 2004, LR 2002):

$\sigma_{E T}=\frac{G J+E I_{W N} \frac{\pi^{2}}{a^{2}}}{I_{N}}$

where $I_{W N}$ and $I_{N}$ are the sectorial moment of inertia and polar moment of inertia about point N, respectively. Given expression for calculation of $I_{N}$ for angle-bar stiffeners in all rule-based equation is as follows:

$I_{N}=\frac{t_{w} h_{w}^{3}}{3}+t_{f} b_{f} h_{w}^{2}$

Comparing Equation (22) with (6), it reveals that in all rule-based equation, second term in Equation (6) is neglected. Given expressions for calculation of $I_{W N}$ for angle-bar stiffeners in some of leading classification society rules are as follows:

- $L R$ (2002); $B V$ (2002):

$I_{W N}=\frac{b_{f}^{3} h_{w}^{2}}{12\left(b_{f}+h_{w}\right)^{2}}\left(t_{f}\left(b_{f}^{2}+2 b_{f} h_{w}+4 h_{w}^{2}\right)+3 t_{w} b_{f} h_{w}\right)$

- GL (2004); CSR for oil Tankers (2006): 
$I_{W N}=\frac{t_{f} b_{f}^{3} h_{w}^{2}}{12} \frac{2.6 t_{w} h_{w}+t_{f} b_{f}}{t_{w} h_{w}+t_{f} b_{f}}$

- $A B S$ (2004):

$I_{W N}=\frac{t_{f} b_{f}^{3} h_{w}^{2}}{12} \frac{3 t_{f} b_{f}+4 t_{w} h_{w}}{t_{f} b_{f}+t_{w} h_{w}}\left(1.7-0.1 \frac{h_{w}}{b_{f}}\right)+\frac{t_{w}^{3} h_{w}^{3}}{36}$

- $D N V(2010)$ :

$I_{W N}=h_{w}^{2}\left(\frac{1}{12} A_{f} b_{f}^{2}+\left(\frac{b_{f}}{2}\right)^{2}\left(\frac{A_{f}}{1+\frac{A_{f}}{A_{w}}}\right)\right)$

\section{Numerical Examples and Discussions}

\subsection{Comparison of sectorial moment of inertia}

For a comparative purpose, sectorial moment of inertia for common angle-bar stiffeners used in ship structure are calculated by Equation (9) and checked against rule-based equations (Tables 1-2).

Table 1: Ratio of sectorial moment of inertia of angle bars calculated by rule-based equation over Equation (9)

\begin{tabular}{|l|l|l|l|l|}
\hline Angle bar & LR (2002), $B V(2002)$ & GL (2004), CSR (2006) & ABS (2004) & DNV (2010) \\
\hline $100 \times 65 \times 7$ & 1 & 0.699 & 1.981 & 1 \\
\hline $100 \times 75 \times 7$ & 1 & 0.705 & 2.063 & 1 \\
\hline $100 \times 50 \times 10$ & 1 & 0.689 & 1.842 & 1 \\
\hline $120 \times 80 \times 8$ & 1 & 0.700 & 1.995 & 1 \\
\hline $125 \times 75 \times 10$ & 1 & 0.696 & 1.937 & 1 \\
\hline $130 \times 65 \times 8$ & 1 & 0.689 & 1.837 & 1 \\
\hline $150 \times 75 \times 9$ & 1 & 0.689 & 1.837 & 1 \\
\hline $150 \times 90 \times 9$ & 1 & 0.696 & 1.935 & 1 \\
\hline $160 \times 80 \times 10$ & 1 & 0.689 & 1.837 & 1 \\
\hline $150 \times 90 \times 12$ & 1 & 0.696 & 1.937 & 1 \\
\hline $180 \times 90 \times 10$ & 1 & 0.689 & 1.836 & 1 \\
\hline $200 \times 100 \times 10$ & 1 & 0.689 & 1.836 & 1 \\
\hline $200 \times 100 \times 12$ & 1 & 0.689 & 1.837 & 1 \\
\hline $200 \times 100 \times 14$ & 1 & 0.689 & 1.838 & 1 \\
\hline $250 \times 90 \times 12$ & 1 & 0.679 & 1.662 & 1 \\
\hline
\end{tabular}

As can be seen, Equation (9) and given equation in LR (2002), and BV (2002) have the same results for angle bars with equal thickness at web and flange. However, for angle bars with unequal thickness of web and flange, there are up to $4 \%$ deviation between LR (2002), and BV (2002) and Equation (9). Given equation in GL (2004), yields about 30\% lower values than Equation (9) for both types of angle bars. Given equation by ABS (2004) gives higher values than Equation (9) for both types of angle bars. Only given equation by DNV (2009) yields exactly the same results as Equation (9). Therefore, in the further analysis DNV (2009) or Equation (9) has been used. 
Table 2: Ratio of sectorial moment of inertia of angle bars calculated by rule-based equation over Equation (9) (unequal thickness)

\begin{tabular}{|l|l|l|l|l|}
\hline Angle bar & $\begin{array}{l}\text { LR (2002), } B V \\
(2002)\end{array}$ & GL (2004), CSR (2006) & ABS (2004) & DNV (2010) \\
\hline $200 \times 90 \times 9 / 14$ & 1.027 & 0.702 & 1.920 & 1 \\
\hline $250 \times 90 \times 9 / 15$ & 1.034 & 0.696 & 1.795 & 1 \\
\hline $250 \times 90 \times 12 / 16$ & 1.011 & 0.688 & 1.731 & 1 \\
\hline $300 \times 90 \times 11 / 16$ & 1.017 & 0.684 & 1.639 & 1 \\
\hline $300 \times 90 \times 13 / 17$ & 1.009 & 0.681 & 1.616 & 1 \\
\hline $350 \times 90 \times 12 / 17$ & 1.014 & 0.679 & 1.535 & 1 \\
\hline $400 \times 100 \times 11.5 / 16$ & 1.012 & 0.678 & 1.512 & 1 \\
\hline $400 \times 100 \times 13 / 18$ & 1.012 & 0.678 & 1.512 & 1 \\
\hline $450 \times 125 \times 11.5 / 18$ & 1.023 & 0.684 & 1.605 & 1 \\
\hline $450 \times 150 \times 11.5 / 15$ & 1.009 & 0.684 & 1.676 & 1 \\
\hline $500 \times 150 \times 11.5 / 18$ & 1.024 & 0.687 & 1.655 & 1 \\
\hline $550 \times 150 \times 12 / 21$ & 1.036 & 0.687 & 1.619 & 1 \\
\hline $600 \times 150 \times 12.5 / 23$ & 1.041 & 0.686 & 1.570 & 1 \\
\hline
\end{tabular}

\subsection{Finite element method}

To demonstrate the accuracy and applicability of the proposed method, a series of finite element method (FEM) eigenvalue analyses are performed. The computer code ANSYS (version 5.6) has been used for this analysis. Flange and web of angle bars are modeled using four-node quadrilateral shell element (SHELL63) with six degrees of freedom per node. To enforce tripping about junction point of web to attached plate and prevent flexural buckling, displacement in $y$ and $z$ directions at the baseline of web are restrained. To ensure tripping of beam without web or flange distortion, rigid web and flange are created. Due to symmetry only half length of stiffeners are modeled. A uniformly distributed normal stress is applied over one end while holding the other end symmetry condition. Fig. 4 shows boundary conditions and applied load in FE model. Fig. 5 depicts buckling deformation of an angle-bar stiffener due to tripping.

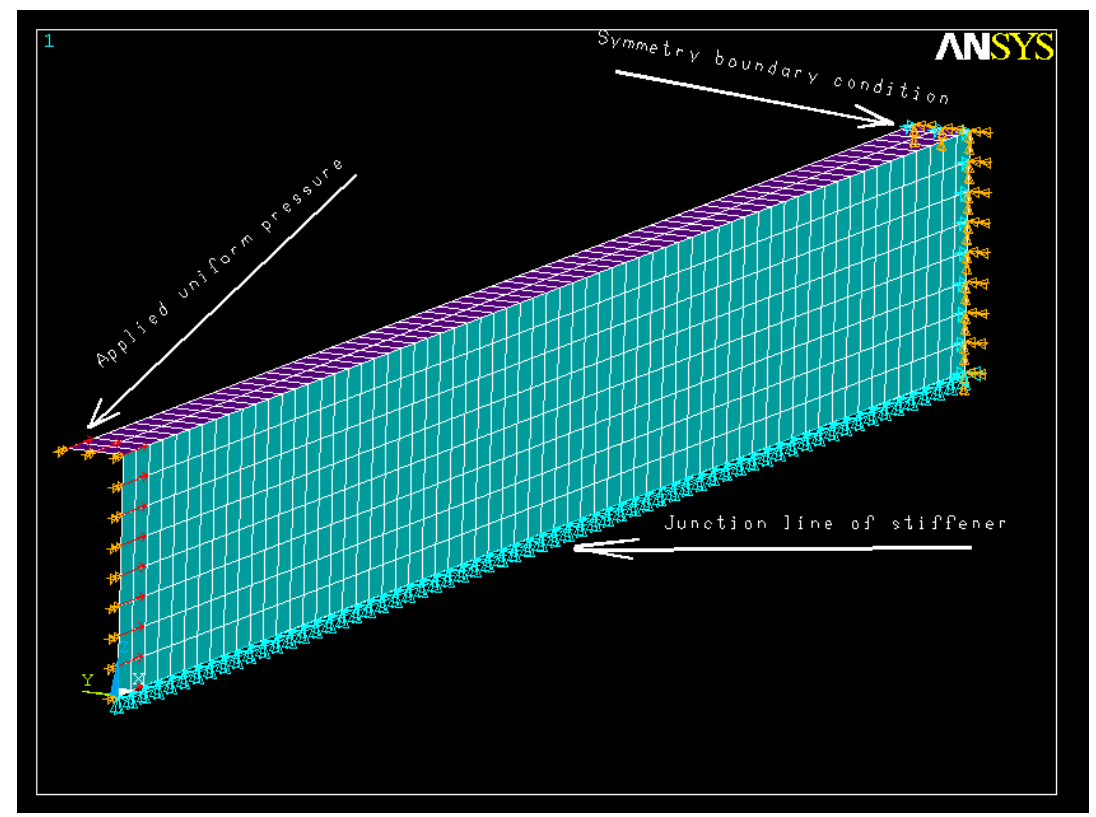

Fig. 4: Boundary conditions and applied load in FE model 


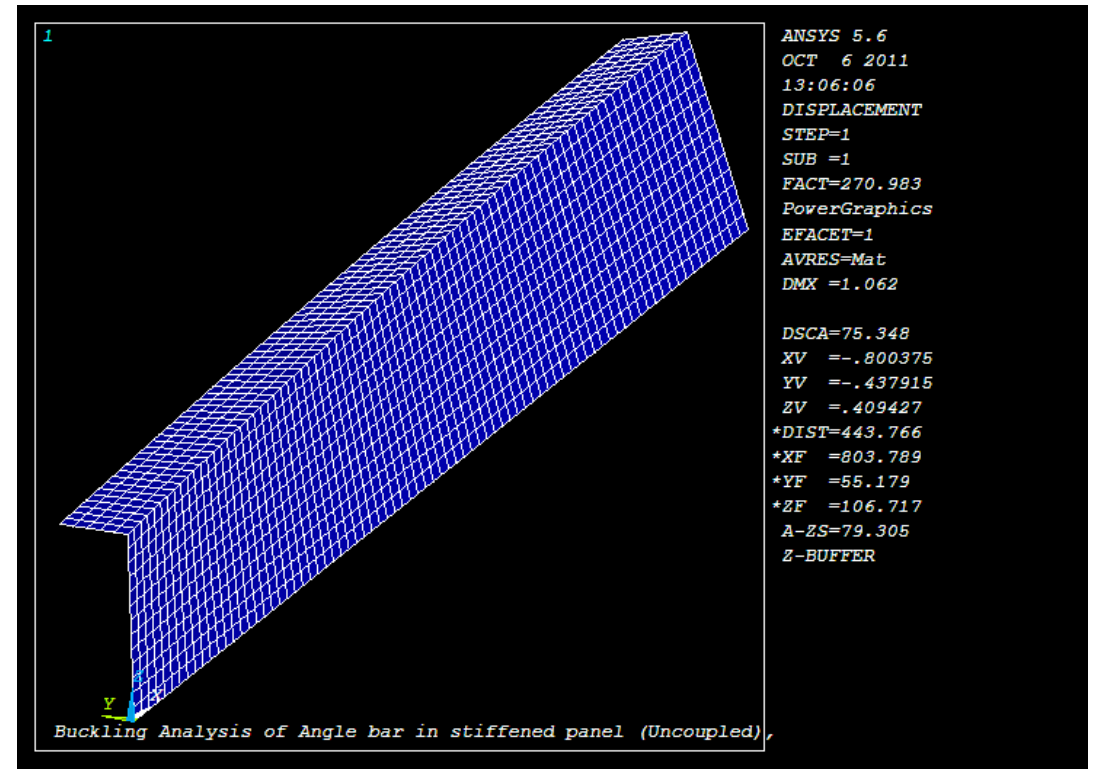

Fig. 5: Buckling deformation of an angle-bar stiffener due to tripping

\subsection{Comparative study on Euler tripping stress and discussions}

Tables 3 and 4, show elastic tripping stress of angle bar stiffeners calculated by different method. Material is considered as mild steel with $E=206 \mathrm{GPa}, v=0.3$, and different angle bars with a length of $3200 \mathrm{~mm}$ has been considered. Results of different methods are presented and compared with FEM.

Table 3: Tripping Euler stress (MPa) calculated by rule-based equation for angle bars in stiffened plate (equal thickness)

\begin{tabular}{|l|l|l|l|l|}
\hline \multirow{2}{*}{ Angle bar } & \multicolumn{2}{|l|}{ Rule-based equation } & Equation (5) & \multirow{2}{*}{ FEM } \\
\cline { 2 - 3 } & Basic & \multicolumn{2}{|l|}{ correction of $I_{N}$} & \\
\hline $100 \times 65 \times 7$ & 347.37 & 317.79 & 318.14 & 317.20 \\
\hline $100 \times 75 \times 7$ & 383.94 & 339.82 & 340.17 & 339.13 \\
\hline $100 \times 50 \times 10$ & 549.84 & 523.66 & 524.44 & 522.92 \\
\hline $120 \times 80 \times 8$ & 390.30 & 357.94 & 358.40 & 357.19 \\
\hline $125 \times 75 \times 10$ & 461.77 & 428.70 & 429.43 & 428.11 \\
\hline $130 \times 65 \times 8$ & 305.86 & 291.29 & 291.79 & 290.93 \\
\hline $150 \times 75 \times 9$ & 338.66 & 322.54 & 323.17 & 322.04 \\
\hline $150 \times 90 \times 9$ & 410.69 & 381.27 & 381.87 & 380.09 \\
\hline $160 \times 80 \times 10$ & 376.30 & 358.38 & 359.16 & 357.75 \\
\hline $150 \times 90 \times 12$ & 537.46 & 498.97 & 500.02 & 496.53 \\
\hline $180 \times 90 \times 10$ & 387.96 & 369.48 & 370.27 & 368.26 \\
\hline $200 \times 100 \times 10$ & 416.67 & 396.83 & 397.61 & 393.33 \\
\hline $200 \times 100 \times 12$ & 468.96 & 446.63 & 447.75 & 444.44 \\
\hline $200 \times 100 \times 14$ & 530.76 & 505.48 & 507.01 & 502.38 \\
\hline $250 \times 90 \times 12$ & 342.44 & 334.93 & 336.22 & 333.33 \\
\hline
\end{tabular}


Table 4: Tripping Euler stress (MPa) calculated by rule-based equation for angle bars in stiffened plate (unequal thickness)

\begin{tabular}{|l|l|l|l|l|}
\hline \multirow{2}{*}{ Angle bar } & \multicolumn{2}{|l|}{ Rule-based } & \multirow{2}{*}{ Equation (5) } & \multirow{2}{*}{ FEM } \\
\cline { 2 - 5 } & basic & correction of $I_{N}$ & \\
\hline $200 \times 90 \times 9 / 14$ & 390.42 & 373.35 & 373.96 & 398.28 \\
\hline $250 \times 90 \times 9 / 15$ & 345.49 & 336.16 & 336.79 & 362.87 \\
\hline $250 \times 90 \times 12 / 16$ & 378.08 & 368.68 & 369.84 & 383.16 \\
\hline $300 \times 90 \times 11 / 16$ & 323.39 & 317.98 & 319.00 & 334.81 \\
\hline $300 \times 90 \times 13 / 17$ & 342.77 & 337.30 & 338.77 & 348.99 \\
\hline $350 \times 90 \times 12 / 17$ & 300.91 & 297.48 & 298.78 & 311.01 \\
\hline $400 \times 100 \times 11.5 / 16$ & 326.17 & 322.74 & 323.95 & 334.63 \\
\hline $400 \times 100 \times 13 / 18$ & 340.43 & 336.86 & 338.41 & 349.32 \\
\hline $450 \times 125 \times 11.5 / 18$ & 498.69 & 491.53 & 492.64 & 515.42 \\
\hline $450 \times 150 \times 11.5 / 15$ & 690.39 & 676.22 & 677.32 & 680.24 \\
\hline $500 \times 150 \times 11.5 / 18$ & 699.60 & 687.54 & 688.62 & 710.96 \\
\hline $550 \times 150 \times 12 / 21$ & 702.5 & 692.39 & 693.57 & 724.67 \\
\hline $600 \times 150 \times 12.5 / 23$ & 696.36 & 688.05 & 689.34 & 719.95 \\
\hline
\end{tabular}

As can be seen neglecting second term of Equation (6) for calculation of polar moment of inertia of angle bars leads to about ten percent overestimation of Euler tripping stress.

\section{Conclusions}

Tripping is one of buckling modes in stiffened plates which should be checked according all classification society rules. Sectorial moment of inertia is a key parameter for calculating Euler tripping stress. Given rulebased expressions for calculation of sectorial moment of inertia of angle-bar stiffeners are scattered. Different rule-based equations for calculation of sectorial moment of inertia of angle-bar stiffeners are investigated, and it is found that some rule-based equations for calculation of sectorial moment of inertia of angle-bar stiffeners are inaccurate. It is also found that in rule-based equation for calculation of polar moment of inertia, one term is neglected which leads up to $10 \%$ over estimation of elastic tripping stress. Euler tripping stress for different angle-bar stiffeners are calculated by energy method and compared with FEM and rule-based expression.

\section{Reference}

ABS (2004): Buckling and Ultimate strength assessment for offshore structures, American Bureau of shipping.

BV (2002): Rules and Regulations for the classification of ships, Pt.B, Sec.4, Bureau Veritas.

CSR for oil Tankers (2006): Part CSR-T Common structural rules for double hull oil tankers.

Danielson, D.A., Kihl, D.P., Hodges, D.H. (1990): Tripping of Thin-Walled Plating Stiffeners in Axial. ThinWalled Struct. 10: 121-142.

DNV (2010): Recommended Practice, DNV-RP-C201, Buckling strength of plated structures. Section 7.5.2, Det Norske Veritas.

GL (2004): Rules and Guidelines, Pt.1, Sec.3F Design Principles, Germanischer LIoyd's.

LR (2002): Rules and Regulations for the classification of ships Pt.3, Ch.4, Sec.7, LIoyd's Register of Shipping.

Rahbar-Ranji, A. (2012): Elastic tripping analysis of angle bars and permanent means of access structures, Ocean Engineering 53: 128-137 doi:10.1016/j.oceaneng.2012.07.008

Timoshenko, S.P., Gere, J.M. (1961): Theory of elastic stability, $2^{\text {nd }}$ ed. Engineering Societies Monograph, NY: McGraw Hill. 